\title{
La evaluación del perfeccionismo: utilidad de la Escala Multidimensional de Perfeccionismo en población española
}

\author{
Ángel Carrasco, Amparo Belloch y Conxa Perpiñá \\ Universidad de Valencia
}

\section{RESUMEN}

La Escala Multidimensional de Perfeccionismo (MPS) de Frost et al. (1990) es uno de los instrumentos con más tradición empírica y mayor influencia en el estudio del perfeccionismo. El objetivo de este trabajo es traducir y validar la MPS para su uso en población española. Para ello, 434 participantes (77\% mujeres; edad media: 23,38 años), completaron la versión española de la MPS junto con cuestionarios de obsesividad, trastornos alimentarios, y preocupaciones ansiosas, para evaluar la validez convergente de la MPS. El análisis de componentes principales (varianza explicada: 56\%), reveló una estructura de 4 factores en lugar de los 6 originales: Miedo a los errores, Influencias paternas, Expectativas de logro y Organización. La consistencia interna de la escala total y sus subescalas fueron excelentes $(\alpha=0,87$ a 0,93$)$. La MPS mostró una validez convergente adecuada a través de su asociación con diferentes medidas de psicopatología, en especial con las referidas a obsesiones.

\section{Palabras Clave}

Perfeccionismo, MPS, trastorno obsesivocompulsivo, trastornos de la conducta alimentaria.

\section{ABSTRACT}

The Multidimensional Perfectionism Scale (MPS; Frost et al. 1990) has been extensively used in empirical research and is one of the most theoretically influential instruments in the research about perfectionism. The aim of the present study has been to translate and validate the MPS for its use in the Spanish population. To this end, 434 participants (77\% women; mean age: 23.38 years), completed the Spanish version of the MPS, together with questionnaires measuring obsessiveness, eating disorders and anxious worries, in order to measure the MPS' convergent validity. A principal component analysis (\% of explained variance: $56 \%$ ) revealed a four-factor structure instead of the originally proposed six dimensions: Fear of mistakes, Parental influences, Achievement expectation, and Organization. Internal consistencies for the scale and its subscales were excellent ( $\alpha$ values ranging from 0.87 to 0.93 ). The MPS showed an adequate convergent validity through its association to measures of psychopathology, especially with obsessivecompulsive disorder instruments.

KEY WORDS

Perfectionism, MPS, obsessive-compulsive disorder, eating disorders.

Esta investigación forma parte del proyecto de investigación SEJ2006/03893-PSIC (Ministerio de Ciencia y Tecnología) de la Acción Especial GVAE-2007/011 (Generalitat Valenciana) (A. Belloch, investigador principal). Los autores agradecen al Dr. R. Frost su permiso para adaptar al castellano la Escala Multidimensional de Perfeccionismo.

Correspondencia: Amparo Belloch Fuster. Departamento de Personalidad, Evaluación y Tratamiento Psicológicos. Facultad de Psicología. Avda. Blasco Ibáñez, 21. 46010 Valencia. E-mail: amparo.belloch@uv.es 


\section{Introducción}

El perfeccionismo ha sido un tema de interés creciente en la investigación psicopatológica durante las últimas tres décadas hasta el punto de ser considerado una variable relevante en numerosos trastornos psicológicos, como en la depresión (Brown y Beck, 2002), los trastornos alimentarios (Fairburn, 1997), el trastorno obsesivo-compulsivo (Frost, Novara y Rhéaume, 2002), la fobia social (Antony, Purdon, Huta y Swinson., 1998) y en síntomas psicosomáticos (Shafran y Mansell, 2001). Los estudios empíricos sobre el perfeccionismo fueron muy escasos hasta la elaboración de los primeros instrumentos de medida que vieron la luz en la década de 1980, vinculados a la teoría cognitiva de la depresión y a la investigación psicométrica de los trastornos de la alimentación (Enns y Cox, 2002). El impulso definitivo a la investigación estuvo vinculado con la publicación a principios de los 90 de dos instrumentos, denominados del mismo modo: Multidimensional Perfectionism Scale (Frost, Marten, Lahart y Rosenblate, 1990; Hewitt y Flett, 1991).

El desarrollo de la investigación sobre el propio constructo de perfeccionismo $y$, consecuentemente, de los instrumentos para su evaluación, ha propiciado la diversificación de las definiciones y/o acepciones de perfeccionismo. En la actualidad existen diferencias importantes en cómo es conceptualizado por los diferentes investigadores, lo que implica que estas diferencias deben tenerse muy en cuenta a la hora de interpretar los resultados de las diversas investigaciones (Flett y Hewitt, 2002). Con todo, Purdon, Antony y Swinson
(1999), afirman que existe un acuerdo generalizado desde el punto de vista teórico acerca de cuáles son los dos componentes básicos del constructo perfeccionismo: en primer lugar, las exigencias personales elevadas, es decir, la existencia de criterios exigentes para definir el éxito personal, y en segundo término la autoevaluación negativa en caso de que no se alcancen esos criterios. Por otro lado, puede afirmarse que el enfoque unidimensional ha ido perdiendo terreno a favor de una concepción que reconoce dimensiones relativamente independientes dentro del mismo constructo, y actualmente nadie duda de que no todos sus componentes son necesariamente negativos.

La Multidimensional Perfectionism Scale de Frost (Frost et al., 1990) ha sido posiblemente el instrumento de medida del perfeccionismo más influyente en psicopatología, tanto desde el punto de vista teórico como por su uso en la investigación aplicada. Los creadores de la escala se basaron en una revisión teórica para elaborar 67 ítems que capturaban lo que consideraron los aspectos más relevantes del perfeccionismo. La depuración sucesiva de esa escala inicial redujo el número inicial de ítems a 35, agrupados en seis dimensiones: 1) Personal standards (Exigencias personales), definida como tendencia a marcarse uno mismo metas excesivamente elevadas y dar un peso excesivo a las mismas en la autoevaluación; 2) Concern over mistakes (Preocupación por los errores), o preocupación excesiva por los errores y el fracaso en diferentes áreas de la vida; 3) Doubts about actions (Dudas sobre acciones), es decir, tendencia a dudar excesivamente sobre la calidad de la propia ejecución; 
4) Parental expectations (Expectativas paternas), entendido como percepción de que los propios padres tienen expectativas elevadas sobre uno; 5) Parental criticism (Críticas paternas), o la percepción de los propios padres como abiertamente críticos; y 6) Organization (Organización), que refiere a un énfasis en la importancia del orden y la organización. De todas ellas, la Preocupación por los errores ha sido señalada por los autores originales como la más claramente vinculada con la presencia de psicopatologías (Frost y DiBartolo, 2002). No obstante, es importante señalar que en la definición de estas dimensiones las consideraciones teóricas tuvieron un peso más importante que los resultados empíricos. Como consecuencia, las investigaciones posteriores no son concordantes a la hora de reproducir estas seis dimensiones propuestas por los autores, lo que lleva a la ausencia de acuerdo sobre cuál es la mejor o más adecuada estructura factorial de la escala.

Las propiedades psicométricas básicas son, a pesar de todo, satisfactorias (Frost et al., 1990; Enns y Cox, 2002), pues tanto la escala completa como sus subescalas muestran valores de consistencia interna entre aceptables y excelentes con valores de a entre 0,70 y 0,95 , según los diversos estudios (cfr., Hewitt y Flet, 1991). Además, muestra una buena validez convergente (Frost, Heiberg, Holt, Mattia y Neubauer, 1993), así como relaciones sustantivas con medidas de psicopatología en diversas investigaciones, y en especial con medidas de trastornos alimentarios (BardoneCone, Wonderlich, Frost, Bulik, Mitchell, Uppala, y Simonich, 2007), depresión y trastorno obsesivo-compulsivo (Sassaroli, Romero, Rug- giero, Mauri, Vinai, y Frost, 2008). Este tipo de estudios refuerzan además la hipótesis de la multidimensionalidad del constructo, que ha recibido un cierto apoyo a partir de un estudio de gemelos (Tozzi, Aggen, Neale, Anderson, Mazzeo, Neale, y Bulik, 2004). Sin embargo, existen dudas sobre su validez de constructo, así como sobre su poder predictivo y la especificidad de las relaciones de sus dimensiones con las diversas formas de psicopatología en las que hipotéticamente juega un papel relevante (Enns y Cox, 2002). En un estudio reciente realizado con población general y tres grupos de pacientes (depresivos, obsesivocompulsivos y con trastornos alimentarios), si bien los pacientes difirieron de la población general, no se constataron diferencias claras entre los tres grupos de pacientes. Además, la dimensión de Preocupación por los errores fue la más claramente vinculada con indicadores de psicopatología en estos tres grupos, y la de Dudas sobre acciones la más específicamente relacionada con obsesión-compulsión y trastornos alimentarios (Sassaroli et al., 2008). Según estos resultados, resulta evidente que las diversas dimensiones del perfeccionismo se asocian a la presencia de psicopatologías, pero la especificidad de tales relaciones teniendo en cuenta los trastornos analizados es dudosa.

En definitiva, la influencia teórica de la Multidimensional Perfectionism Scale de Frost et al. (1990), así como su amplio uso en la investigación psicopatológica, hacen de esta escala un instrumento imprescindible en el estudio del perfeccionismo, así como la utilidad del constructo evaluado en la investigación de las variables que intervienen en 
la génesis y/o el mantenimiento de diversos trastornos mentales.

Este trabajo tiene por objetivo traducir y adaptar al castellano la MPS de Frost et al. (1990) así como proporcionar datos relevantes sobre su validez y posible utilidad en el contexto hispanohablante. Por ello, además de examinar sus propiedades psicométricas se planteará una aproximación a la validez convergente de la escala, así como su relación con diferentes medidas de psicopatología en población general.

\section{Método}

\section{Participantes}

En este estudio se utilizaron dos muestras, en dos fases consecutivas tal y como se explica en el apartado de procedimiento. La primera incluía a 100 personas, todas ellas estudiantes de los dos últimos cursos de la licenciatura de Psicología de la Universidad de Valencia. La mayoría eran mujeres (80\%), con una edad media de 21 años $(D T=1,5)$, y sin antecedentes ni historia actual de trastorno mental diagnosticado. La segunda muestra estaba formada por 334 personas provenientes de la comunidad, si bien una parte importante la formaban estudiantes universitarios (60\%) y el resto eran familiares o conocidos. Había 254 mujeres (76\%) y 80 hombres (24\%). La edad media de los participantes era de 23,38 años $(D T=3,89)$, y la mayoría (80\%) tenía estudios universitarios. No había diferencias en nivel de estudios, sexo, ni edad entre los sujetos de ambas muestras. Los participantes colaboraron en todos los casos de forma voluntaria sin recibir compensación económica a cambio.

\section{Instrumentos}

Escala Multidimensional de Perfeccionismo (MPS). Traducción al castellano de la Multidimensional Perfectionism Scale de Frost (Frost et al., 1990). Cuestionario de 35 ítems con una escala de respuesta entre el 1 y el 5 . Está compuesta por seis subescalas correspondientes a las dimensiones del perfeccionismo postuladas por sus autores y descritas en la introducción: Exigencias personales, Preocupación por los errores, Dudas sobre acciones, Expectativas paternas, Críticas paternas, y Organización. La escala fue traducida al castellano por el primer autor del trabajo y revisada y retrotraducida al inglés por las otras autoras, así como por una traductora oficial anglohablante, especializada en textos de contenido psicológico. La MPS fue completada por la totalidad de la muestra (434 personas). En el Apéndice se ofrece una relación de los items que forman el cuestionario.

Obsessive-Compulsive Inventory - Revised (OCl-R) (Foa, Huppert, Leiberg, Langner, Kichic, Hajcak, y Salkovskis, 2002). Instrumento de autoinforme de 18 ítems diseñado para evaluar el malestar asociado a síntomas obsesivo-compulsivos. La escala de respuesta oscila entre 0 (nada de malestar) y 4 (muchísimo malestar).. Se ha utilizado la versión española de Fullana, Tortella, Caseras, Andión, Torrubia y Mataix, 2004), que reproduce la estructura factorial original (Lavado, Obsesiones, Acumulación, Orden, Comprobación, Neutralización) y tiene buenas propiedades psicométricas. En el presente estudio, este cuestionario fue completado por la totalidad de la muestra $(n=434)$.

Clark- Beck Obsessive Compulsive Inventory (C-BOCl). -Inventario Obsesivo-Compulsivo de 
Clark y Beck- (Clark y Beck, 2002). Instrumento de cribaje para evaluar la frecuencia de síntomas obsesivos y compulsivos. Está compuesto de 25 ítems con dos subescalas (obsesiones y compulsiones) en una escala Likert de 4 puntos (0: "nunca"- 3: "con mucha frecuencia"). Las propiedades psicométricas han sido evaluadas en una muestra formada por pacientes con diagnóstico de trastorno obsesivo compulsivo, pacientes clínicos sin problemas obsesivos y población general. Los datos muestran una buena validez de constructo, una sólida validez convergente y una modesta validez discriminante (Clark, Antony, Beck, Swinson y Steer, 2005). Se ha utilizado la adaptación y validación a la población española (Belloch, Reina, García-Soriano \& Clark, 2009) que obtuvo una consistencia interna (a) para las subescalas de obsesiones y compulsiones de 0,89 y 0,81 respectivamente, $y$ una estabilidad test-retest (intervalo de 15 días, evaluada mediante el coeficiente de correlación intraclase, $\mathrm{CCl}$ ) de 0,88 y 0,84 para cada subescala respectivamente. En el presente estudio, este cuestionario fue completado por la segunda muestra ( $n=334$ personas).

Cuestionario de Preocupación de Pensilvania (Penn State Worry Questionnaire, PSWQ; Meyer, Miller, Metzger y Borkovec, 1990). Instrumento autoinformado que evalúa la tendencia a preocuparse por diferentes aspectos de la vida cotidiana. Consta de 16 ítems, que se puntúan según una escala tipo Likert de 1 ("nada típico en mi") a 5 ("muy típico en mi"). Se utilizó la adaptación española de Sandín y Chorot (1991). Este cuestionario fue completado por la totalidad de la muestra $(n=434)$.

The Eating Attitudes Test (EAT-26) -Cuestio- nario de las actitudes hacia la comida- (Garner, Olmsted, Bohr y Garfinkel, 1982). Versión abreviada del EAT-40 (Garner y Garfinkel, 1979). Consta de 26 ítems que evalúan un amplio rango de actitudes y comportamientos relacionados con los trastornos alimentarios, principalmente con la anorexia nerviosa. Cada ítem se puntúa en una escala tipo Likert que va desde "nunca" hasta "siempre". La puntuación total indica gravedad de los síntomas, pero no tiene validez diagnóstica. Este instrumento, además de la puntuación total consta de las escalas "Dieta”, “Bulimia y preocupación por el alimento" $y$ "Control oral". El instrumento tiene una consistencia interna adecuada $(a=0,79)$ (Garner y Garfinkel, 1979). Para este trabajo se ha empleado la versión y adaptación española realizada por Castro, Toro, Salamero y Guimerá (1991). Este cuestionario fue completado por la segunda muestra ( $n=334$ personas).

Obsessive Compulsive Personality Disorder Subscale: Personality Diagnostic Questionnaire (PDQ-4) -Subescala del Trastorno Obsesivo Compulsivo de la Personalidad, del Cuestionario Diagnóstico de Personalidad- (Hyler, 1994). El Personality Diagnostic Questionnaire -4 es un instrumento de autoinforme diseñado para evaluar trastornos de personalidad según los criterios del DSM-IV. Consta de 12 escalas de trastornos de personalidad. En este estudio sólo se ha empleado la escala de trastorno obsesivo compulsivo de la personalidad (TOCP), que consta de 8 afirmaciones con dos opciones de respuesta $(V / F)$ en función de si la persona considera que le son o no aplicables. Cada ítem puntúa en un criterio diagnóstico propuesto por el DSM-IV para el TOCP. La consistencia in- 
terna de la escala de TP obsesivo compulsivo es bastante moderada. De hecho, se ha informado de un valor de $a=0,34$ en la adaptación del cuestionario a población española (Calvo, Caseras, Gutiérrez y Torrubia, 2002), versión que es la utilizada en este trabajo. En el presente estudio, este cuestionario fue completado por la segunda muestra ( $n=334$ personas).

Inventario de Creencias Obsesivas Revisado (ICO-R) (Belloch, Cabedo, Morillo, Lucero, y Carrió, 2003; Cabedo, Belloch, Morillo, Giménez, y Carrió, 2004; Giménez, Morillo, Belloch, Carrió, y Cabedo, 2004; Luciano, Belloch, Morillo, y Garcia-Soriano, 2006). Es un instrumento de autoinforme diseñado para evaluar las diferentes áreas de creencias disfuncionales hipotéticamente implicadas en el desarrollo y/o mantenimiento del TOC, que se compone de 50 ítems a responder en escalas Likert de 7 puntos (desde 1: "completamente en desacuerdo" a 7: "completamente de acuerdo"). Los ítems se agrupan en 8 escalas: Responsabilidad excesiva; Importancia de los pensamientos; Fusión Pensamiento Acción (FPA)-probabilidad; FPA-moral; Importancia de controlar los pensamientos; Sobreestimación del peligro; Intolerancia a la incertidumbre y Perfeccionismo. Para los propósitos de este trabajo únicamente se utiliza la subescala de Perfeccionismo. Este cuestionario fue completado por la segunda muestra ( $n=334$ personas).

\section{Procedimiento}

Como se ha dicho, una parte de la muestra, compuesta por 100 personas, estaba íntegramente formada por estudiantes de Psicología de la Universidad de Valencia. Estos completaron, además de la MPS, el PSWQ y el OCI-R junto con sus datos demográficos, de forma grupal y en horario docente, sin recibir compensación económica ni académica a cambio.

El resto de la muestra (334 personas) se obtuvo siguiendo la estrategia de "bola de nieve", para lo que se recabó la colaboración voluntaria de estudiantes de Psicología de la Universidad de Valencia. Para ello, se les ofreció un seminario sobre los pensamientos intrusos en los trastornos obsesivos y alimentarios, así como sobre el propósito del presente estudio. Se les instruyó sobre el manejo de todos los cuestionarios que se han descrito, así como sobre su finalidad y valoración. Posteriormente, se les pidió que pasaran este protocolo a 4 o 5 familiares y/o amigos, de entre $14 \mathrm{y}$ 40 años, de forma individual y estando presentes mientras los participantes los completaban. Los participantes no debían tener historia de trastorno mental, no haber recibido o estar recibiendo tratamiento psicológico o farmacológico durante los dos últimos años, y no tener dificultades para leer y comprender las instrucciones escritas.

\section{Resultados}

\section{Estructura factorial}

En primer lugar, se comprobó mediante las pruebas estadísticas pertinentes que los datos eran susceptibles de ser sometidos a un análisis factorial. En el test de Kaiser-Meyer-Olkin (KMO) se obtuvo un índice de 0,93, y el resultado de la prueba de esfericidad de Bartlett fue $X^{2}(595)=8597, p<0,001$. En consecuencia, se procedió a la realización de los análisis que se comentan a continuación. 
El análisis factorial exploratorio (componentes principales, rotación varimax) produjo, según el scree test, dos soluciones factoriales interpretables: una con 6 factores y otra con 4. No obstante, los valores del análisis de cuatro factores eran más adecuados, en especial por lo que se refiere por un lado, al contenido de los items y a su interpretación, y por otro, a los autovalores obtenidos: del cuarto al quinto factor el autovalor pasaba de 1,71 a 1,18, descendiendo muy suavemente a partir de dicho factor. En consecuencia, se decidió optar por una solución de 4 factores, que explicaba el $56 \%$ de la varianza. En la tabla 1 se muestra la composición de cada factor después de la rotación varimax.

En el primer factor se incluyeron 11 ítems, todos ellos procedentes de las subescalas originales Preocupación por errores y Dudas sobre acciones. Esta última subescala está representada íntegramente en el factor, mientras que la de Preocupación aporta 7 de sus 9 ítems. Se incluyen aquí los aspectos más negativos del perfeccionismo, por lo que siguiendo a Purdon et al., (1999) lo hemos denominado "Miedo a los errores". El segundo factor estuvo formado por 9 ítems, todos ellos procedentes de las dos dimensiones originales que hacen referencia a los estándares familiares en la génesis del perfeccionismo: Expectativas paternas y Críticas paternas. En consecuencia, denominamos este factor "Influencias paternas". El tercer factor se compuso con 9 ítems, e incluyó los siete ítems de la escala original Exigencias personales además de otros dos (el 13y el 18) correspondientes a la escala original de Preocupación por errores. El contenido de estos dos últimos ítems hace referencia a la competitividad y la comparación con el rendimiento ajeno a la hora de evaluar el propio. Aunque ambos mantenían saturaciones superiores a 0,40 en el primer factor, se decidió incluirlos en el tercero para no desviarse de los criterios empíricos a la hora de decidir la composición factorial. Llamamos a este factor "Expectativas de logro" siguiendo la sugerencia de Harvey, Pallant y Harvey (2004). El último factor incluyó los seis ítems de la subescala original "Organización", por lo que se mantuvo la misma denominación.

\section{Consistencia interna}

Para analizar la consistencia interna del cuestionario, se utilizó el coeficiente a de Cronbach, tanto para la escala total como para cada una de las subescalas derivadas del análisis factorial antes comentado. El valor de a para la escala total fue de 0,93 . Los obtenidos para los cuatro factores fueron los siguientes: Miedo a errores: $a=0,88$; Influencias paternas: $a=0,90$; Expectativas de logro: $a=0,87$; Organización : $a=0,89$.

Con el fin de comparar estos valores con los derivados de la estructura original de 6 dimensiones, se procedió a calcular también el valor de alfa de las mismas. Los valores obtenidos en este caso fueron los siguientes: Preocupación por errores: $a=0,88$; Exigencias personales: $a$ $=0,84$; Expectativas paternas: $a=0,87$; Críticas paternas: $a=0,77$; Dudas: $a=0,76$; y Organización: $a=0,89$. Según estos resultados, tanto en la solución de 4 factores obtenida en este estudio, como en la original de 6 dimensiones, los datos sobre la consistencia interna del instrumento son entre buenos y excelentes, si bien 
Tabla 1

Cargas factoriales y coeficientes de comunalidad de la Escala Multidimensional de Perfeccionismo (análisis de componentes principales, rotación varimax) $(n=434)$

\begin{tabular}{|c|c|c|c|c|c|c|}
\hline \multirow[b]{2}{*}{ Ítem } & \multirow[b]{2}{*}{ Subescala original } & \multicolumn{4}{|c|}{ Factores } & \multirow[b]{2}{*}{$h^{2}$} \\
\hline & & Miedo a errores & $\begin{array}{l}\text { Influencias } \\
\text { Paternas }\end{array}$ & $\begin{array}{c}\text { Expectativas } \\
\text { De Logro }\end{array}$ & Organización & \\
\hline 9 & Preocupación Errores & 0,53 & 0,11 & 0,41 & 0,02 & 0,47 \\
\hline 10 & Preocupación Errores & 0,41 & 0,13 & 0,36 & 0,09 & 0,33 \\
\hline 14 & Preocupación Errores & 0,56 & 0,20 & 0,40 & 0,03 & 0,52 \\
\hline 17 & Dudas & 0,44 & 0,18 & 0,34 & 0,24 & 0,41 \\
\hline 21 & Preocupación Errores & 0,69 & 0,25 & 0,26 & 0,04 & 0,62 \\
\hline 23 & Preocupación Errores & 0,68 & 0,23 & 0,21 & $-0,03$ & 0,57 \\
\hline 25 & Preocupación Errores & 0,60 & 0,26 & 0,36 & 0,04 & 0,57 \\
\hline 28 & Dudas & 0,62 & 0,05 & 0,12 & 0,22 & 0,46 \\
\hline 32 & Dudas & 0,58 & 0,30 & $-0,05$ & 0,22 & 0,48 \\
\hline 33 & Dudas & 0,58 & 0,28 & 0,07 & 0,16 & 0,46 \\
\hline 34 & Preocupación Errores & 0,63 & 0,20 & 0,26 & 0,03 & 0,52 \\
\hline 1 & Expectativas Paternas & $-0,02$ & 0,70 & 0,23 & 0,09 & 0,55 \\
\hline 3 & Criticas Paternas & 0,23 & 0,60 & 0,12 & 0,04 & 0,43 \\
\hline 5 & Criticas Paternas & 0,34 & 0,50 & 0,03 & $-0,01$ & 0,37 \\
\hline 11 & Expectativas Paternas & 0,09 & 0,73 & 0,26 & 0,09 & 0,62 \\
\hline 15 & Expectativas Paternas & 0,24 & 0,75 & 0,25 & 0,02 & 0,69 \\
\hline 20 & Expectativas Paternas & 0,16 & 0,77 & 0,28 & 0,02 & 0,70 \\
\hline 22 & Criticas Paternas & 0,37 & 0,73 & $-0,01$ & $-0,07$ & 0,68 \\
\hline 26 & Expectativas Paternas & 0,13 & 0,83 & 0,00 & $-0,01$ & 0,70 \\
\hline 35 & Criticas Paternas & 0,26 & 0,68 & 0,04 & $-0,02$ & 0,53 \\
\hline 4 & Exigencias Personales & 0,40 & 0,09 & 0,54 & 0,04 & 0,47 \\
\hline 6 & Exigencias Personales & 0,10 & 0,06 & 0,65 & 0,27 & 0,51 \\
\hline 12 & Exigencias Personales & 0,25 & 0,16 & 0,71 & 0,14 & 0,61 \\
\hline 13 & Preocupación Errores & 0,46 & 0,14 & 0,57 & 0,04 & 0,55 \\
\hline 16 & Exigencias Personales & $-0,31$ & $-0,01$ & 0,61 & 0,35 & 0,60 \\
\hline 18 & Preocupación Errores & 0,43 & 0,15 & 0,62 & 0,06 & 0,59 \\
\hline 19 & Exigencias Personales & 0,31 & 0,27 & 0,63 & 0,13 & 0,59 \\
\hline 24 & Exigencias Personales & 0,19 & 0,19 & 0,56 & 0,14 & 0,41 \\
\hline 30 & Exigencias Personales & 0,25 & 0,20 & 0,62 & 0,31 & 0,58 \\
\hline 2 & Organización & 0,11 & 0,07 & 0,25 & 0,73 & 0,62 \\
\hline 7 & Organización & 0,08 & 0,03 & 0,10 & 0,78 & 0,65 \\
\hline 8 & Organización & 0,07 & 0,00 & 0,16 & 0,85 & 0,75 \\
\hline 27 & Organización & 0,07 & $-0,02$ & 0,18 & 0,85 & 0,76 \\
\hline 29 & Organización & 0,25 & 0,14 & 0,07 & 0,62 & 0,47 \\
\hline 31 & Organización & $-0,00$ & $-0,10$ & 0,09 & 0,80 & 0,66 \\
\hline
\end{tabular}

$h^{2}=$ coeficiente de comunalidad. En negrita cursiva se indican los items que fueron asignados a cada factor. 
son ligeramente superiores los obtenidos con la versión tetrafactorial que se deriva de nuestros datos.

\section{Relaciones entre dimensiones}

Se calcularon las correlaciones (Pearson) entre las diferentes subescalas del cuestionario derivadas factorialmente, así como entre éstas y la puntuación total. Las correlaciones con la puntuación total fueron significativas $(p \leq 0,01)$ en todos los casos. Las más elevadas fueron las obtenidas entre los factores Miedo a errores $(r=0,72)$ y Expectativas de logro $(r=0,70)$, y la más baja la de Influencias paternas $(r=0,49)$ y Organización $(r=0,35)$. En cuanto a las asociaciones de los factores entre sí, todos los coeficientes fueron significativos con un valor de $p \leq 0,01$, con la única excepción de la relación entre Influencias paternas y Expectativas de logro $(r=0,12 ; p=0,05)$. La subescala de Organización fue la que mantuvo unos coeficientes menores de correlación con las otras tres y con la puntuación total.

También se calcularon las correlaciones entre las subescalas correspondientes a las seis dimensiones originales del cuestionario, haIlando resultados muy similares. Únicamente la correlación entre Organización y Críticas paternas no resultó significativa, mientras que el resto alcanzaron un valor de $p \leq 0,01$.

\section{Datos normativos}

En la tabla 2 se muestran los datos descriptivos obtenidos para la puntuación total en la MPS así como en las 4 subescalas derivadas factorialmente y las 6 escalas originales.

No se hallaron diferencias significativas entre sexos en la puntuación total ni en ninguna de las

Tabla 2

Datos descriptivos de la Escala Multidimensional de Perfeccionismo (MPS) y sus subescalas (versión de 4 factores y versión original)

\begin{tabular}{lccccc}
\hline MPS & $\begin{array}{c}\text { Mujeres } \\
(n=334)\end{array}$ & $\begin{array}{c}\text { Hombres } \\
(n=100)\end{array}$ & $\begin{array}{c}\text { Total } \\
(n=434)\end{array}$ & $\begin{array}{c}\text { Asimetría } \\
(n=434)\end{array}$ & $\begin{array}{c}\text { Percentiles } \\
25 / 50 / 75\end{array}$ \\
\hline Puntuación Total & $70,71(18,62)$ & $73,18(21,13)$ & $71,28(19,23)$ & 0,97 & $56 / 67 / 82$ \\
\hline \multicolumn{7}{c}{ Factores/ Subescalas del presente estudio } \\
\hline Miedo a errores & $19,05(6,72)$ & $19,88(7,22)$ & $19,24(6,84)$ & 1,18 & $14 / 18 / 23$ \\
Influencias Paternas & $14,14(6,13)$ & $14,83(7,16)$ & $14,30(6,38)$ & 1,82 & $10 / 12 / 16$ \\
Expectativas de logro & $19,05(6,75)$ & $20,32(6,74)$ & $19,34(6,76)$ & 0,82 & $14 / 18 / 24$ \\
Organización* & $18,47(5,20)$ & $18,15(4,74)$ & $18,40(5,09)$ & 0,23 & $15 / 18 / 22$ \\
\hline \multicolumn{7}{c}{ Subescalas originales } \\
\hline Preocupación por Errores & $14,38(5,72)$ & $15,38(6,17)$ & $14,61(5,83)$ & 1,42 & $10 / 13 / 17$ \\
Exigencias Personales & $15,63(5,37)$ & $16,77(5,44)$ & $15,89(5,40)$ & 0,70 & $12 / 15 / 19$ \\
Expectativas Paternas & $8,54(4,09)$ & $9,05(4,65)$ & $8,66(4,23)$ & 1,56 & $5 / 7 / 10$ \\
Críticas Paternas & $5,60(2,50)$ & $5,78(2,86)$ & $5,64(2,59)$ & 2,17 & $4 / 4 / 6$ \\
Dudas sobre acciones & $8,09(3,18)$ & $8,05(3,18)$ & $8,08(3,18)$ & 0,93 & $6 / 8 / 10$ \\
\hline
\end{tabular}

Los datos se expresan como media (DT).

* Esta subescala es idéntica a la de la versión original por lo que no se reproduce de nuevo bajo el rótulo de subescalas originales (véase el texto para más explicación). 
subescalas. A pesar de la escasa variabilidad en edad de la muestra utilizada, se han constatado algunas relaciones significativas entre esta variable y las puntuaciones en dos de las subescalas del MPS derivadas factorialmente en el presente estudio: Miedo a Errores $(r=-0,12)$ y Expectativas de logro $(r=-0,10)$. Asimismo, se encontraron relaciones entre la edad y las subescalas Preocupación por errores $(r=-0,10)$, Exigencias personales $(r=-0,10)$ y Dudas $(r=-0,14)$ de la composición original del instrumento. No obstante, atendiendo a los tamaños de los coeficientes de correlación, estas asociaciones fueron de escasa entidad ( $R^{2}=0,02$ en el mejor de los casos).

\section{Validez convergente}

Con el fin de examinar las asociaciones del perfeccionismo con las medidas de obsesividad, trastornos alimentarios, y preocupaciones ansiosas (PSWQ), se realizaron análisis de correlaciones entre la MPS y las mencionadas medidas. En la Tabla 3 se muestran los resultados obtenidos.

Por lo que se refiere a las asociaciones de la MPS con la escala de Perfeccionismo del Inventario de Creencias Obsesivas se obtuvo un coeficiente alto, según el criterio de Cohen (1988), lo que avala la validez convergente entre ambos cuestionarios $\left(R^{2}=0,33\right)$. En cuanto

Tabla 3

Correlaciones de Pearson entre la Escala Multidimensional de Perfeccionismo y el resto de instrumentos

\begin{tabular}{|c|c|c|c|c|c|}
\hline \multirow[b]{2}{*}{ Cuestionarios } & \multicolumn{5}{|c|}{ Escala Multidimensional de Perfeccionismo (MPS) } \\
\hline & Total & Miedo errores & Influencias Paternas & $\begin{array}{c}\text { Expectativas } \\
\text { de logro }\end{array}$ & Organización \\
\hline ICO: Perfeccionismo ${ }^{a}$ & $0,58^{* *}$ & $0,53^{* *}$ & $0,35^{* *}$ & $0,54^{* *}$ & $0,39 * *$ \\
\hline PDQ: TOCPa & $0,49^{* *}$ & $0,47^{* *}$ & $0,32 * *$ & $0,45^{* *}$ & $0,28 * *$ \\
\hline $\mathrm{PSWQ}^{\mathrm{b}}$ & $0,35^{* *}$ & $0,42^{* *}$ & $0,14^{* *}$ & $0,28^{* *}$ & $0,21 * *$ \\
\hline \multicolumn{6}{|c|}{ Clark-Beck Obsessive Compulsive Inventory (C-BOCI) } \\
\hline Total $^{\mathrm{a}}$ & $0,52^{* *}$ & $0,61 * *$ & $0,34 *$ & $0,38^{* *}$ & $0,28 * *$ \\
\hline Obsesiones $^{\mathrm{a}}$ & $0,53^{* *}$ & $0,62^{* *}$ & $0,36^{* *}$ & $0,40^{* *}$ & $0,25^{* *}$ \\
\hline Compulsiones $^{\mathrm{a}}$ & $0,43^{* *}$ & $0,50^{* *}$ & $0,26^{* *}$ & $0,29 * *$ & $0,28^{* *}$ \\
\hline \multicolumn{6}{|c|}{ Obsessive-Compulsive Inventory-Revised (OCI-R) } \\
\hline Total $^{b}$ & $0,46^{* *}$ & $0,48^{* *}$ & $0,33^{* *}$ & $0,25^{*}$ & 0,15 \\
\hline Lavar $^{\mathrm{b}}$ & $0,29 * *$ & $0,36^{* *}$ & 0,17 & 0,12 & 0,12 \\
\hline Comprobarb & $0,32^{* *}$ & $0,26 * *$ & $0,30 *$ & 0,19 & 0,11 \\
\hline Ordenar $^{\mathrm{b}}$ & $0,38^{* *}$ & $0,28 * *$ & 0,18 & $0,25^{*}$ & $0,34^{* *}$ \\
\hline Obsesiones $^{b}$ & $0,42^{* *}$ & $0,46^{* *}$ & $0,28^{* *}$ & $0,30 * *$ & 0,02 \\
\hline Acumular ${ }^{b}$ & $0,32^{* *}$ & $0,44^{* *}$ & 0,19 & 0,15 & 0,02 \\
\hline Neutralizar ${ }^{\mathrm{b}}$ & $0,37^{* *}$ & $0,42^{* *}$ & $0,28^{* *}$ & 0,12 & 0,07 \\
\hline \multicolumn{6}{|c|}{ Eating Attitudes Test (EAT) } \\
\hline Total $^{a}$ & $0,33^{* *}$ & $0,32 * *$ & $0,21^{* *}$ & $0,28 * *$ & $0,22 * *$ \\
\hline Dieta $^{a}$ & $0,27^{* *}$ & $0,26 * *$ & $0,15^{* *}$ & $0,22 * *$ & $0,20 * *$ \\
\hline Bulimia $^{a}$ & $0,29 * *$ & $0,32^{* *}$ & $0,21 * *$ & $0,25^{* *}$ & 0,10 \\
\hline Control oral ${ }^{\mathrm{a}}$ & $0,29 * *$ & $0,24 * *$ & $0,20 * *$ & $0,25^{* *}$ & $0,22 * *$ \\
\hline
\end{tabular}

ICO: Inventario de Creencias Obsesivas; PDQ-TOCP: Personality Disorders Questionnaire-Escala de Trastorno Obsesivo-Compulsivo de Personalidad; PSWQ = Penn State Worry Questionnaire.

${ }^{*} p<0,05 ;{ }^{* *} p<0,01 ;{ }^{a} n=334 ;{ }^{b} n=434$ 
a las relaciones entre la MPS con las medidas de obsesividad es decir, los cuestionarios PDQ, $\mathrm{CBOCl}$ y $\mathrm{OCl}-\mathrm{R}$, el rango del tamaño de las correlaciones osciló entre moderado y alto (criterios de Cohen, 1988). Especialmente elevadas fueron las asociaciones con las subescalas de obsesiones tanto del $\mathrm{CBOCl}$ como del OCl-R. Sin embargo, las asociaciones con la medida de patología alimentaria (EAT) presentaron coeficientes de tamaño pequeño. Lo mismo puede decirse con respecto a la medida de preocupaciones ansiosas (PSWQ). Por lo que respecta a las asociaciones de las subescalas de la MPS con el resto de las medidas, las correlaciones más elevadas se constataron en el caso de Miedo a los errores, mientras que Organización fue la subescala que presentó menores o más bajas asociaciones.

\section{Discusión}

La mayoría de los investigadores coinciden en la actualidad en que el perfeccionismo es un constructo multidimensional. La influencia de la Escala Multidimensional de Perfeccionismo de Frost es, en gran medida, responsable de esta concepción. Y esta influencia se basa en el acierto de sus creadores a la hora de incluir en su instrumento contenidos diversos relacionados con el constructo y de unirlos en dimensiones sugerentes teóricamente y fecundas para la investigación psicopatológica. El objetivo de este estudio ha sido examinar la viabilidad de tales dimensiones en una muestra española, así como ofrecer datos descriptivos de la mencionada escala y su asociación con medidas de creencias obsesivas (perfeccionismo), obsesividad, patología alimentaria, y preocupación ansiosa.
Los diferentes estudios publicados sobre la validación de la MPS han hallado, generalmente, estructuras factoriales que no se corresponden con las dimensiones propuestas por los autores de la escala. El resultado más repetido de los análisis factoriales, al menos con muestras no clínicas, ha sido una solución tetrafactorial que se corresponde básicamente con la obtenida en el presente estudio (Harvey et al., 2004; Hawkins, Watt y Sinclair, 2006; Stöber, 1998; Stumpf y Parker, 2000). Es decir, el mantenimiento sin variaciones sustanciales de las subescalas originales Exigencias personales y Organización, y la fusión en un solo factor de las dimensiones Preocupación por errores y Dudas sobre acciones, por un lado, y de Expectativas y Críticas paternas por otro. La dimensión que une las subescalas de Dudas y Preocupación por los errores se considera como la más propiamente negativa de la escala, y su fusión es un resultado sólido e interesante teóricamente, ya que une la preocupación por cometer errores en las actividades cotidianas a una dimensión, la de duda acerca de esas mismas acciones, que contiene ítems inspirados en la patología obsesivocompulsiva. La unión de los factores que se refieren a las críticas y las expectativas paternas es también un resultado habitual. Si bien los resultados son muy similares, el nombre y la interpretación de los factores varían ligeramente a través de los diferentes estudios que han sido objeto de importantes debates (Harvey et al., 2004).

Las diferencias entre las diversas soluciones tetrafactoriales halladas se deben, en nuestra opinión, al tratamiento que reciben los ítems 
problemáticos, bien por tener saturaciones elevadas en más de un factor, bien por saturar de forma predominante en un factor que no se corresponde con las dimensiones propuestas originalmente. Khawaja y Armstrong (2005) optaron por elaborar una versión reducida de la escala dejando fuera los 9 ítems que saturaban por encima de 0,30 en más de un factor. Hawkins et al. (2006) excluyeron los ítems 16 y 18 por el mismo motivo. Stöber (1998) y Harvey et al. (2004) informan también de problemas con el ítem 18 ya que, como en el presente trabajo, saturaba de forma más elevada en el factor que corresponde de forma general con la dimensión de Exigencias personales que con la unión de las dimensiones de Preocupación y Dudas. Stöber (1998) optó por mantenerlo junto con los demás ítems de Preocupación, mientras que Harvey y sus colaboradores lo incluyeron con los ítems de Exigencias, que es la solución que hemos adoptado en este estudio.

La saturación más elevada de los ítems 13 ("Si alguien hace una tarea en el colegio/trabajo mejor que yo, siento como si hubiera fracasado totalmente en esa tarea") y 18 ("Odio no llegar a ser el mejor en todo lo que hago") en la escala de Expectativas de logro, como se ha hallado en este trabajo, se debe probablemente a características de la muestra. Parece que en una muestra predominantemente universitaria como la nuestra, la competitividad y el afán de ser mejor que los demás se asocian en gran medida a unas expectativas elevadas de rendimiento (que puede incluir, en un ambiente competitivo, el superar a los compañeros), y no a una preocupación excesiva por las re- percusiones de los errores. Sin embargo, esto también indica que probablemente la escala no esté reflejando componentes tan "positivos" como sugieren algunos autores. En esta misma línea, es posible que el hecho de que la mayoría de las muestras utilizadas para los estudios de validación estén compuestas de estudiantes afecte no sólo las cargas factoriales de ciertos ítems, sino a la misma estructura factorial obtenida en estas investigaciones. De hecho, Purdon et al. (1999) hallaron que una muestra de tres factores era más satisfactoria en una muestra clínica. Por otro lado, tanto estos últimos autores como Stumpf y Parker (2000) afirman que el uso de las seis subescalas originales es defendible desde el punto de vista psicométrico, útil desde el punto de vista teórico si se desea una descripción detallada del constructo, e incluso recomendable para su uso en investigación psicopatológica. Por estos motivos, hemos optado por incluir algunos datos acerca de las subescalas originales.

Además de la inestabilidad de su estructura factorial, otro problema de la MPS que afecta a su validez de constructo es la cuestión de si la subescala de Organización debería incluirse o no en el cálculo de la puntuación total (Hawkins et al., 2006). En nuestro estudio, la correlación entre este factor y el resto de la escala fue de tamaño medio, según los criterios de Cohen (1988). Este dato, y la consideración de que el resto de factores, aunque más relacionados empíricamente entre sí, tampoco forman una unidad desde el punto de vista conceptual, nos parece suficiente para mantener estos ítems dentro de la puntuación total de la escala. 
Por lo que se refiere a la fiabilidad de la escala (consistencia interna), los datos obtenidos con la estructura tetrafactorial fueron ligeramente mejores que con la original de seis factores. Estos resultados están en línea con los obtenidos por otros estudios de validación (Enns y Cox, 2002), e indican que la escala es consistente desde el punto de vista psicométrico.

En cuanto a la validez convergente, la MPS mantiene una correlación elevada con la escala de perfeccionismo del ICO. La magnitud de esta correlación indica que ambas medidas están más relacionadas entre sí que con cualquier otra medida de psicopatología obsesiva o alimentaria de las incluídas en el estudio, lo que avala la convergencia de ambos instrumentos en la evaluación del constructo. Al mismo tiempo, el hecho de que la asociación entre ambos no sea superior al 33\% (atendiendo al coeficiente de determinación), indica que ambas medidas evalúan aspectos similares, pero no idénticos, del perfeccionismo. Las correlaciones obtenidas entre la MPS y diversas medidas de psicopatología obsesiva proporcionan evidencia adicional sobre la validez y la utilidad en la investigación psicopatológica de la escala, y están en línea con los resultados obtenidos por otros autores. Las magnitudes de las correlaciones entre la MPS y dos medidas de sintomatología obsesivo-compulsiva ( $\mathrm{CBOCl}$ y $\mathrm{OCl}-\mathrm{R})$ son moderadas y similares a otros estudios (por ejemplo, Frost et al., 1990), tal y como ha sido además revisado por Frost y DiBartolo (2002). Es de destacar que la sintomatología obsesivo-compulsiva que más se relaciona en este estudio con el perfeccionismo es la propiamente obsesiva, por encima de la compulsiva y de dimensiones del OCl-R como la comprobación o la acumulación que se han postulado como especialmente cercanas a este constructo. Es posible que este resultado esté relacionado de alguna manera con las características de la muestra. Además, el factor de Miedo a los errores es el más estrechamente relacionado con este tipo de psicopatología y el de Organización el que lo está en menor grado. Las correlaciones con el PSWQ son sustancialmente menos elevadas que las observadas para los cuestionarios de obsesividad. Si bien la existencia de una correlación moderada con esta medida de preocupación ansiosa invita a estudiar en mayor profundidad la conexión entre ambos constructos, también apunta a que la asociación entre perfeccionismo y obsesividad no es exclusivamente debida a la mediación de la ansiedad. Por último, la MPS también mantiene correlaciones significativas pero menores con la sintomatología alimentaria valorada con el EAT. De nuevo son las escalas Expectativas de logro y, sobre todo, Miedo a errores, las que mantienen las correlaciones más elevadas con la sintomatología alimentaria. Nuevamente el perfeccionismo es un factor a tener en cuenta en el estudio de este grupo de psicopatologías (Bardone-Cone et al., 2007) y la MPS parece un instrumento válido para investigar a este respecto.

El presente estudio tiene limitaciones para responder a los objetivos que nos habíamos planteado. En primer lugar, la utilización de una muestra de estudiantes universitarios no permite llegar a conclusiones generalizables a población general sobre la estructura factorial del cuestionario. Futuras investigaciones, 
preferentemente con muestras clínicas o provenientes de la comunidad (no universitarias), deberán darnos más pistas sobre el carácter y contenidos de la multidimensionalidad del constructo. En segundo lugar, es necesario explorar más a fondo las relaciones de la escala con otras medidas del perfeccionismo, si bien la validez convergente de este instrumento parece estar fuera de duda. Por último, la investigación del perfeccionismo deberá ampliarse a otro tipo de muestras, patologías e instrumentos.

A pesar de lo anteriormente señalado, pensamos que la versión española de la Multidimensional Perfectionism Scale indica que este cuestionario es fiable y válido para explorar el constructo del perfeccionismo y su papel en diferentes tipos de psicopatología. Por ello, creemos que su traducción al español supone una aportación útil y que su uso en muestras españolas es aconsejable en la investigación sobre el perfeccionismo $y$, en especial, las vinculaciones de este constructo con psicopatologías vinculadas al espectro obsesivo-alimentario.

\section{Bibliografía}

Antony, M. M., Purdon, C. L., Huta, V. \& Swinson, R. P. (1998). Dimensions of perfectionism across the anxiety disorders. Behaviour Research and Therapy, 36, 1143-1154.

Bardone-Cone, A. M., Wonderlich, S. A., Frost, R. O., Bulik, C. M., Mitchell, J. E., Uppala, S. \& Simonich, H. (2007). Perfectionism and eating disorders: Current status and future directions. Clinical Psychology Review, 27, 384-405.

Belloch, A., Cabedo, E., Morillo, C., Lucero, M. \& Carrió, C (2003). Development of an instru- ment to assess dysfunctional beliefs in the obsessive-compulsive disorder: Preliminary results of the Obsessive Beliefs Inventory. International Journal of Clinical and Health Psychology, 3, 235-250.

Belloch, A., Reina, N., García-Soriano, G. \& Clark, D. (2009). El Inventario Clark-Beck de ObsesiónCompulsión (C-BOCl): Validación para su uso en población española. Revista de Psicopatología y Psicología Clínica, 14, 95-105.

Brown, G. P. \& Beck. A. T. (2002). Dysfunctional attitudes, perfectionism and models of vulnerability to depression. En G. L. Flett \& P. L. Hewitt (Eds.), Perfectionism. Theory, research and treatment (pp. 231-251). Washington, DC: American Psychological Association.

Cabedo, E., Belloch, A., Morillo, C., Giménez, A. \& Carrió, C. (2004). Strength of dysfunctional beliefs in relation to the increased obsessionality. International Journal of Clinical and Health Psychology, 4, 465-479.

Calvo, N., Caseras, X., Gutierrez, F. \& Torrubia, R. (2002). Adaptación española del Personality Diagnostic Questionnarie-4+ (PDQ-4+). Actas españolas de psiquiatría, 30, 7-13.

Castro, J., Toro, J., Salamero, M. \& Guimerá, E. (1991). The eating attitudes test: Validation on the Spanish version. Psychological Assessment, 2, 175-190.

Clark, D. A. \& Beck, A. T. (2002). Manual for the Clark-Beck Obsessive Compulsive Inventory. San Antonio, TX: Psychological Corporation.

Clark, D. A., Antony, M. M., Beck, A. T., Swinson, R. P. \& Steer, R.A. (2005). Screening for Obsessive and Compulsive Symptoms: Validation of the Clark-Beck Obsessive-Compulsive Inventory. Psychological Assessment, 17, 132-143.

Cohen, J. (1988). Statistical power analysis for the behavioral sciences (2nd ed.). Nueva York: Academic Press.

Enns, M. W. \& Cox, B. J. (2002). The nature and assessment of perfectionism: A critical analysis. En G. L. Flett y P. L. Hewitt (Eds.), 
Perfectionism. Theory, research and treatment (pp. 33-62). Washington, DC: American Psychological Association.

Fairburn, C. G. (1997). Eating disorders. En D. M. Clark \& C. G. Fairburn (Eds.), Science and practice of cognitive behaviour therapy (pp. 204-241). Oxford: Oxford University Press.

Flett, G. L. y Hewitt, P. L. (2002). Perfectionism and maladjustment: an overview of theoretical, definitional, and treatment issues. En G. L. Flett \& P. L. Hewitt (Eds.), Perfectionism. Theory, research and treatment (pp. 5-31). Washington, DC: American Psychological Association.

Foa, E. B., Huppert, J. D., Leiberg, S., Langner, R., Kichic, R., Hajcak, G. \& Salkovskis, P. M. (2002). The Obsessive-Compulsive Inventory: development and validation of a short version. Psychological Assessment, 14, 485-496.

Frost, R. O. y DiBartolo, P. M. (2002). Perfectionism, anxiety and obsessive-compulsive disorder. En G. L. Flett \& P. L. Hewitt (Eds.), Perfectionism. Theory, research and treatment (pp. 341-371). Washington, DC: American Psychological Association.

Frost, R. O., Heimberg, R. G., Holt, C. S., Mattia, J. I. \& Nuebauer, M. (1993). A comparison of two measures of perfectionism. Personality and Individual Differences, 14, 119-126.

Frost, R. O., Novara, C. \& Rhéaume, J. (2002). Perfectionism in obsessive compulsive disorder. En R. O. Frost \& G. Steketee (Eds.), Cognitive approaches to obsessions and compulsions: Theory, assessment and treatment (pp. 91-105). Oxford: Elsevier.

Frost, R. O., Marten, P., Lahart, C. \& Rosenblate, R. (1990). The dimensions of perfectionism. Cognitive Therapy and Research, 14(5), 449-468.

Fullana, M. A., Tortella-Feliu, M., Caseras, X., Andión, Ó., Torrubia, R. \& Mataix-Cols, D. (2005). Psychometric properties of the Spanish version of the Obsessive-Compulsive Inventory-Revised in a non-clinical sample. Journal of Anxiety Disorders, 19, 893-903.
Garner D. M. \& Garfinkel P. E. (1979) The Eating Attitudes Test: an index of the symptoms of anorexia nervosa. Psychological Medicine, 9, 273-249.

Garner, D. M., Olmsted, M. P., Bohr, I. \& Garfinkel, P. E. (1982). The Eating Attitudes Test: psychometric features and clinical correlates. Psychological Medicine, 12, 871-878.

Giménez, A., Morillo, C., Belloch, A., Carrió, C. \& Cabedo, E. (2004). Creencias disfuncionales asociadas a síntomas obsesivo-compulsivos: una aproximación a la especificidad. Revista de Psicopatología y Psicología Clínica, 9, 1-18.

Harvey, B., Pallant, J. \& Harvey, D. (2004). An evaluation of the factor structure of the Frost Multidimensional Perfectionism Scale. Educational and psychological measurement, 64, 1007-1018.

Hawkins, C. C., Watt, H. M. G. \& Sinclair, K. E. (2006). Psychometric properties of the Frost Multidimensional Perfectionism Scale with Australian adolescent girls. Clarification of multidimensionality and perfectionist typology. Educational and psychological measurement, 66, 1001-1022.

Hewitt, P. L. \& Flett, G. L. (1991). Perfectionism in the Self and Social Contexts: Conceptualization, Assessment and Association with Psychopathology. Journal of Personality and Social Psychology, 60, 456-470.

Hyler, S. E. (1994). Personality Diagnostic Questionnaire-4 (PDQ-4). Nueva York: New York State Psychiatric Institute.

Khawaja, N. G. \& Armstrong, K. A. (2005). Factor structure and psychometric properties of the Frost Multidimensional Perfectionism Scale: Developing shorter versions using an Australian sample. Australian Journal of Psychology, 57, 129-138.

Luciano, J. V., Belloch, A., Morillo, C. \& Garcia-Soriano, G. (2006). Confirmatory factor analysis of the Obsessive Beliefs Inventory-Revised (OBSIR). EABCT 2006 Congress, Paris.

Meyer, T. J., Miller, M. L., Metzger R. L. \& Borko- 
vec, T.D. (1990). Development and validation of the Penn State Worry Questionnaire. Behaviour Research and Therapy, 28, 487-495.

Purdon, C., Antony, M. M. \& Swinson, R. P. (1999). Psychometric properties of the Frost Multidimensional Perfectionism Scale in a clinical anxiety disorders sample. Journal of Clinical Psychology, 55, 1271-1286.

Reina, N., Belloch, A., Morillo, C. \& Clark, D. (2007). The Clark-Beck Obsessive-Compulsive Inventory: Validation of the Spanish version as a screening instrument for the obsessivecompulsive disorder. Poster. V World Congress of Behavioral and Cognitive Therapies, Barcelona.

Sassaroli, S., Romero, L. J., Ruggiero, G., Mauri, M. C., Vinai, P., \& Frost, R. (2008). Perfectionism in depression, obsessive-compulsive disorder and eating disorders. Behaviour Research and Therapy, 46, 757-765.

Sandín, B. \& Chorot, P. (1991). Adaptación española del Penn State Worry Questionnaire. Madrid: UNED.

Shafran, R. \& Mansell, W. (2001). Perfectionism and psychopathology: A review of research and treatment. Clinical Psychology Review, 21(6), 879-906.

Stöber, J. (1998). The Frost Multidimensional Perfectionism Scale revisited: More perfect with four (instead of six) dimensions. Personality and Individual Differences, 24(4), 481-491.

Stumpf, H. \& Parker, W. D. (2000). A hierarchical structure analysis of perfectionism and its relations to other personality characteristics. Personality and Individual Differences, 28, 837-852.

Tozzi, F., Aggen, S. H., Neale, B. M., Anderson, C. B., Mazzeo, S. E., Neale, M. C. \& Bulik, C. M. (2004). The structure of perfectionism: A twin study. Behavior Genetics, 34(5), 483-494. 


\section{Apéndice}

Escala Multidimensional de Perfeccionismo

(Frost, Marten, Lahart \& Rosenblate, 1990)

1. Mis padres me pusieron metas muy altas

2. Para mí, es muy importante que todo esté organizado

3. De niño me castigaban por no hacer las cosas perfectas

4. Si no me pongo las metas más altas, seguramente acabaré siendo un segundón

5. Mis padres nunca intentaron entender mis errores

6. Para mí es importante ser muy competente en todo lo que hago

7. Soy una persona cuidadosa

8. Intento ser organizado

9. Si fracaso en el trabajo/estudios, soy un fracasado

10. Debería sentirme mal si cometo un error

11. Mis padres querían que fuera el mejor en todo

12. Me pongo metas más altas a mí mismo que la mayoría de la gente

13. Si alguien hace una tarea en el colegio/trabajo mejor que yo, siento como si hubiera fracasado totalmente en esa tarea

14. Fracasar en parte es tan malo como fracasar en todo

15. En mi familia sólo se acepta un rendimiento sobresaliente

16. Se me da muy bien concentrar mis esfuerzos en alcanzar una meta

17. Incluso cuando hago algo con mucho cuidado, a menudo me parece que no está bien del todo
18. Odio no llegar a ser el mejor en todo lo que hago

19. Tengo metas extremadamente altas

20. Mis padres esperaban de mí la excelencia, lo mejor

21. Seguramente la gente me apreciará menos si cometo un error

22. Nunca me he sentido capaz de lograr lo que mis padres esperaban de mí

23. Si no hago las cosas tan bien como los demás, significa que soy un ser humano inferior

24. Los demás parecen conformarse con metas más bajas que las mías

25. Si no hago las cosas siempre bien, la gente no me respetará

26. Mis padres siempre han tenido expectativas más altas sobre mi futuro que yo mismo

27. Intento ser una persona cuidadosa

28. Normalmente tengo dudas sobre las pequeñas cosas que hago cada día

29. La pulcritud es muy importante para mí

30. Espero rendir mejor en mis tareas diarias que la mayoría de la gente

31. Soy una persona organizada

32. Tiendo a retrasarme en mi trabajo porque repito las cosas una y otra vez

33. Me cuesta mucho tiempo hacer algo "bien"

34. Cuanto menos errores cometa, más gustaré a los demás

35. Nunca me he sentido capaz de llegar a alcanzar el nivel de mis padres 
